\title{
Article
}

\section{An Enhanced ELISPOT Assay for Sensitive Detection of Antigen-Specific T Cell Responses to Borrelia burgdorferi}

\section{Chenggang Jin ${ }^{1}$, , Diana R. Roen ${ }^{1}$, Paul V. Lehmann ${ }^{2}$ and Gottfried H. Kellermann ${ }^{3}$}

1 Department of Immunology, Pharmasan Labs, Inc., Osceola, WI 54020, USA;

E-Mail: diana.roen@pharmasan.com

2 Cellular Technology Limited, Shaker Heights, OH 44122, USA;

E-Mail:paul.lehmann@immunospot.com

3 NeuroScience, Inc., Osceola, WI 54020, USA; E-Mail: gottfried.kellermann@neurorelief.com

* Author to whom correspondence should be addressed; E-Mail: chenggang.jin@ pharmasan.com;

Tel.: +1-715-294-2144; Fax: +1-715-294-3921.

Received: 11 July 2013; in revised form: 30 August 2013 / Accepted: 4 September 2013 /

Published: 13 September 2013

\begin{abstract}
Lyme Borreliosis is an infectious disease caused by the spirochete Borrelia burgdorferi that is transmitted through the bite of infected ticks. Both B cell-mediated humoral immunity and $\mathrm{T}$ cell immunity develop during natural Borrelia infection. However, compared with humoral immunity, the $\mathrm{T}$ cell response to Borrelia infection has not been well elucidated. In this study, a novel $\mathrm{T}$ cell-based assay was developed and validated for the sensitive detection of antigen-specific $\mathrm{T}$ cell response to B. burgdorferi. Using interferon- $\gamma$ as a biomarker, we developed a new enzyme-linked immunospot method (iSpot Lyme ${ }^{\mathrm{TM}}$ ) to detect Borrelia antigen-specific effector/memory $\mathrm{T}$ cells that were activated in vivo by exposing them to recombinant Borrelia antigens ex vivo. To test this new method as a potential laboratory diagnostic tool, we performed a clinical study with a cohort of Borrelia positive patients and healthy controls. We demonstrated that the iSpot Lyme assay has a significantly higher specificity and sensitivity compared with the Western Blot assay that is currently used as a diagnostic measure. A comprehensive evaluation of the $\mathrm{T}$ cell response to Borrelia infection should, therefore, provide new insights into the pathogenesis, diagnosis, treatment and monitoring of Lyme disease.
\end{abstract}

Keywords: Borrelia infection; T cells; interferon- $\gamma$; ELISPOT 


\section{Introduction}

Lyme disease, caused by infection with the spirochete Borrelia burgdorferi, is an emerging infectious disease in the United States that has become an important public health problem [1-3]. The Centers for Disease Control and Prevention (CDC) reported about 32,500 new cases in 2011 [4], though it is estimated that the actual number might be 10 -fold higher, making Lyme disease an epidemic larger than AIDS, West Nile Virus, and Avian Flu combined. Only a fraction of these cases are being diagnosed and treated, due to an unclear history, equivocal manifestations, inaccurate or insensitive laboratory clinical tests, and underreporting [5]. These undiagnosed and untreated patients may develop chronic infection or late stage Lyme disease such as chronic Lyme arthritis [6,7] and chronic Lyme neuroborreliosis [8,9] which can be devastating in some cases.

The diagnosis of Lyme disease is based primarily on recognizing a characteristic clinical picture [10]. Diagnostic tests for detection of either B. burgdorferi itself, or of the ensuing immune response to it have so far been unreliable. Both $\mathrm{B}$ cell and $\mathrm{T}$ cell immunity develop during a natural infection with B. burgdorferi [11,12]. Detection of the specific antibody response against $B$. burgdorferi is utilized conventionally in aiding the clinical diagnosis of Lyme disease. The standard two-tier tests used to detect specific antibodies to B. burgdorferi include an enzyme-linked immunosorbent assay (ELISA) and a Western Blot assay (WB) [13]. However, the limitation of these assays is that they have low sensitivity and specificity, frequently producing false negative and false positive results. For example, nearly $30 \%$ of results from a Western Blot IgM test are false positive [14]. Furthermore, Borrelia-specific antibodies cannot be detected at the early stage of the infection, and a subgroup of Lyme patients lack detectable Borrelia-specific antibodies [15-17], in both cases providing a false negative result. Borrelia-specific T cell immunity has not yet been studied sufficiently due to the lack of highly sensitive and specific $\mathrm{T}$ cell-based assays that would be suited for the clinical laboratory. Several attempts have been made to study $\mathrm{T}$ cell reactivity against Borrelia, but the results were not consistent from different studies [18-20]. There is increasing evidence, however, that $\mathrm{T}$ cell assays have potential advantages over antibody-based assays in the detection of Borrelia infections. Firstly, patients with erythema chronicum migrans (ECM), a clinical manifestation of B. burgdorferi infection, displayed specific $\mathrm{T}$ cell responses before antibodies to this organism become detectable by ELISA [21,22] and Lastavica et al. reported a case in which seroconversion did not occur until 18 months after the onset of the illness [23]. Secondly, a number of patients who received antibiotics for ECM had low or undetectable levels of anti-Borrelia antibodies suggesting that the antibody response can be decreased or aborted by early antibiotic intervention [24]. Thirdly, antibody titers often drop to levels below the cutoff value for positivity by ELISA, in particular for untreated subjects or patients with chronic Borrelia infection. Fourth, changes in IgM/IgG titers and ratios cannot be used to monitor progress and treatment of Borrelia infection since they may stay constant for as long as 20 years $[25,26]$. Thus, there is a definite need for complementary $\mathrm{T}$ cell assays that may help overcome the aforementioned shortcomings of serological assays for diagnosing and monitoring the progress and treatment of Borrelia infection.

The enzyme-linked immunospot assay (ELISPOT) has emerged as a superior method for assessment of the magnitude and the quality of $\mathrm{T}$ cell immunity. It enumerates at the single cell level the frequency and cytokine signature of activated antigen-specific $\mathrm{T}$ cells [27,28]. The sensitivity of ELISPOT for 
detecting cytokine producing T cells is 20 to 200 fold higher than that of ELISA or flow cytometry-based intracellular staining [29]. The ELISPOT technology has proven to be extremely sensitive in detecting even low frequencies of antigen reactive $T$ cells and has been approved by the FDA for use in the diagnosis of tuberculosis [30,31]. Here, we explore the potential application of our newly developed Lyme ELISPOT assay, iSpot Lyme, as a diagnostic tool for the detection of Lyme Borreliosis.

\section{Materials and Methods}

\subsection{Isolation of Human Peripheral Blood Mononuclear Cells}

Blood donors were either healthy adults without known inflammatory conditions or history of Borrelia infection, or subjects with clinically diagnosed Lyme disease. All individuals whom we classified as Lyme patients met the CDC surveillance definition of Lyme disease, including clinical signs and symptoms, history of possible exposure to infected blacklegged ticks, with or without a positive antibody response to B. burgdorferi by ELISA and Western Blot, interpreted according to CDC and the Infectious Disease Society of America (IDSA) criteria [32,33]. In addition, non-Lyme control patients with other, specified clinical complications were studied including patients diagnosed with Fibromyalgia, Mononucleosis, Rheumatoid Arthritis and Chronic Fatigue Syndrome. These non-Lyme control patients were from low risk areas of Borrelia infection (States ND, MT, UT and AZ) as defined by the CDC. Written informed consent was obtained from all study subjects. Peripheral blood mononuclear cells (PBMC) were separated from acid citrate dextrose (ACD)-treated whole blood using Leucosep tubes (Greiner Bio-One North America, Inc, NC, USA) according to the manufacturer's instruction. The cell concentration was adjusted to $2.5 \times 10^{6} \mathrm{PBMC} / \mathrm{mL}$ in CTL Test Plus Medium (Cellular Technology Limited, OH, USA). The cells were kept at room temperature and seeded into the ELISPOT assay $24 \mathrm{~h}$ after the blood draw. For the study of inter-assay precision, cryopreserved PBMC from one blood draw were used to avoid biological variation of the test sample.

\subsection{ELISPOT Assays with PBMC}

All PBMC samples were assayed using the human IFN- $\gamma$ ImmunoSpot kit by Cellular Technology Limited (OH, USA) per the manufacturer's instruction. The iSpot Lyme test is made available through Pharmasan Labs, Inc. Briefly, the PBMC were plated into anti-IFN- $\gamma$ antibody pre-coated 96-well plates at 250,000 cells per well. The PBMC were then stimulated with $10 \mu \mathrm{g} / \mathrm{mL}$ of a proprietary combination of recombinant (r) Borrelia antigens purchased from DIARECT AG (Freiberg, Germany). A signal enhancer was added concurrently with the rBorrelia antigens and incubated with the PBMC. All culture conditions (negative control, positive control and $\mathrm{r}$ Borrelia antigen stimulation) were tested in triplicate. The PBMC were incubated for $18-24 \mathrm{~h}$ at $37{ }^{\circ} \mathrm{C}, 9 \% \mathrm{CO}_{2}$. The resulting ELISPOTs were analyzed using the CTL S6 Ultimate-V Analyzer (CTL, OH, USA) and are reported as IFN- $\gamma$ Spot Forming Units (SFU). The difference between the iSpot Lyme and the conventional ELISPOT was in the composition of Borrelia antigens and in the use of a signal enhancer in the iSpot Lyme assay. The conventional ELISPOT assay followed the identical protocol to the iSpot Lyme assay, but used unenhanced test medium with the rBorrelia antigens OspC and VlsE, whereas the iSpot Lyme assay used enhanced medium with a proprietary combination of rBorrelia antigens DbpA, OspC, p100, and VlsE. 


\subsection{Measurement of IFN- $\gamma$ Concentration in PBMC Supernatants}

The concentrations of IFN- $\gamma$ in the supernatant from rBorrelia antigen stimulated PBMC were determined using the Bio-Plex suspension array system according to the manufacturer's instructions (Bio-Rad, Hercules, CA, USA). Briefly, supernatants were collected from 96-well plates containing PBMC that were stimulated overnight with rBorrelia antigen, and frozen at $-80{ }^{\circ} \mathrm{C}$ until use. The thawed supernatant samples were incubated in 96-well filter plates at room temperature for $30 \mathrm{~min}$ with antibodies chemically coupled to fluorescent-labeled microbeads. After three washes, premixed detection antibodies were added to each well and incubated for $30 \mathrm{~min}$. Following three washes, premixed streptavidin-phycoerythrin was added to each well and incubated for $10 \mathrm{~min}$. Finally, the beads were washed three times and resuspended with $125 \mu \mathrm{L}$ of assay buffer. The plates were read on a Bio-Plex 200 reader and data were processed and analyzed by using Bio-Plex Manager Software 6.0 (Bio-Rad, Hercules, CA, USA). Values with coefficient of variation (\% CV) above 30 were excluded from the standard curve.

\subsection{Western Blot Assay}

Western Blot assays were performed on patient serum samples by using Borrelia Western Blot IgG and IgM kits (Trinity Biotech, Carlsbad, CA, USA) following the manufacturer's instruction. Briefly, aliquots $(20 \mu \mathrm{L})$ of undiluted serum samples were added to channels containing the test strips and $2 \mathrm{~mL}$ of dilution buffer. Antigens on membranes of this kit were separated by the manufacturer. The IgG kit includes the following 13 bands: p18, p23, p28, p30, p31, p34, p39, p41, p45, p58, p60, p66, and p93; The IgM kit included the following 3 bands: p23, p39, and p14. The strips were scanned using BLOTrix Reader (Frankfurt, Germany). Visualization of specific protein bands indicated the presence of serum IgG or IgM antibodies against B. burgdorferi-derived antigens. Samples were classified as positive or negative in accordance with the criteria established by CDC.

\subsection{Statistical Analysis}

Receiver Operating Characteristic Analysis (ROC) was used to evaluate the accuracy of the tests. The sensitivity was plotted on the $\mathrm{y}$ axis, and the false positive rate (1-specificity) was plotted on the $\mathrm{X}$ axis. For this purpose, the ELISPOT results of 80 healthy people and 25 Lyme patients were studied. The nonparametric Spearman's test was used to determine correlations. The nonparametric Mann-Whitney U test was used to compare ELISPOT results between healthy controls, Lyme patients and non-Lyme patients. A $p$-value of $<0.05$ was considered statistically significant. The analyses were done by GraphPad Prism 5.0 analysis software (La Jolla, CA, USA).

\section{Results and Discussion}

\subsection{Enhanced Detection of Borrelia-Specific Reactive T Cells by the iSpot Lyme Assay}

It is well documented that both humoral and cellular immune responses develop in Borrelia infection. Assessment of both the function and the frequency of Borrelia-specific T cells is crucial for evaluating the cellular immune response to, and diagnosis of Borrelia infection [22,34]. Due to the 
clonal expansion (proliferation) of antigen-specific $\mathrm{T}$ cells in vivo during an immune response, the presence of increased frequencies of Borrelia antigen-specific effector/memory $\mathrm{T}$ cells in peripheral blood suggests prior infection/exposure to Borrelia [35,36]. To establish the frequencies of Borrelia-specific effector/memory T cells in PBMC, we performed ELISPOT assays to measure the numbers of $\mathrm{T}$ cells that secreted IFN- $\gamma$ upon stimulation ex vivo by rBorrelia antigens. PBMC were isolated from both Borrelia positive patients and healthy controls. The cells were plated at 250,000 cells per well and stimulated with recombinant (r) Borrelia antigen for 18 to $24 \mathrm{~h}$, followed by the detection of the IFN- $\gamma$ secreted by the individual T cells resulting in "spots". The numbers of spot forming units (SFU) were counted by an automated ImmunoSpot reader. To measure antigen-triggered $\mathrm{T}$ cell function, we tested the PBMC in a conventional ELISPOT assay and the enhanced Lyme ELISPOT assay (iSpot Lyme assay) in parallel, with a medium that has signal enhancing properties for

T cells, CTL Test Plus. Both the conventional ELISPOT and the iSpot Lyme assay were compared for their sensitivity in detection of Borrelia-specific effector/memory T cells. The results are summarized in Figure 1. Clearly, the newly developed iSpot Lyme assay significantly increased the sensitivity for detecting Borrelia-specific T cells (Figure 1A, $p=0.001$ ). More importantly, the iSpot Lyme assay increased the detection of Borrelia-specific $\mathrm{T}$ cells in Borrelia positive samples, without increasing non-specific spots in healthy controls and the medium control background (Figure 1B\&C). In addition, the spot size distribution was also analyzed and compared between the conventional ELISPOT and the iSpot Lyme assay permitting us to compare the amount of IFN- $\gamma$ produced by the T cells under both conditions. As shown in Figure 1D, the spot sizes in the conventional and the enhanced assay showed the normal distribution that is characteristic of the cytokine signature of T cells [37] and there was no size difference between the spots elicited by the two methods. Therefore, the data suggests that our iSpot Lyme assay specifically increases the number of Borrelia-reactive T cells that secrete IFN- $\gamma$ but does not change the IFN- $\gamma$ productivity of such T cells at the single cell level.

The above results suggest that the iSpot Lyme assay is a highly sensitive in vitro assay for the detection of specific T cell immunity to Borrelia infection. However, since IFN- $\gamma$ is secreted by both recently activated $\mathrm{T}$ effector cells and resting memory $\mathrm{T}$ cells, the iSpot Lyme assay cannot distinguish between active Borrelia infection and prior exposure. There is currently no standard laboratory test to distinguish active Borrelia infection from prior exposure [38].

\subsection{Evaluation of the Sensitivity and Specificity of the iSpot Lyme Assay as a Diagnostic Test}

As the iSpot Lyme assay proved to be a more sensitive tool to detect the Borrelia-specific T cells compared with the conventional ELISPOT assay, we next explored if the iSpot Lyme assay could be used as a laboratory T cell-based diagnostic test for Borrelia infection. For this purpose, PBMC were isolated from 80 healthy controls that had not been exposed to Borrelia (HC), 25 patients with clinically diagnosed Lyme disease (LD) and 23 non-Lyme patients (NLP) who had clinical symptoms similar to Lyme disease. As shown in Figure 2A, the iSpot Lyme assay clearly distinguished the Lyme disease patients from healthy controls and non-Lyme patients, in both cases with a significance level of $p<0.0001$. To further determine the performance of the iSpot Lyme assay, we analyzed the sensitivity, specificity, the positive predictive value (PPV) and the negative predictive value (NPV) using Receiver Operating Characteristic Analysis (ROC). In this study, the iSpot Lyme assay had a 
sensitivity of $84 \%$ vs. $67 \%$, a specificity of $94 \%$ vs. $76 \%$, a PPV of $81 \%$ vs. $48 \%$, and a NPV of $95 \%$ vs. $86 \%$ for conventional ELISPOT, respectively (Figure 2B\&C). The cutoff value was also determined by ROC as 25 SFU per well for the iSpot Lyme assay. Overall, the ROC analysis suggests that the iSpot Lyme assay fulfills the criteria for a reliable diagnostic laboratory test for Borrelia infection with an area under the curve value (AUC) of 0.943 vs. 0.68 for the conventional ELISPOT.

Figure 1. Comparison of detection of Borrelia-specific $\mathrm{T}$ cells in peripheral blood by the iSpot Lyme assay and conventional ELISPOT assay. (A) The frequency of rBorrelia antigen-induced IFN- $\gamma$ spot was established under both conditions in peripheral blood mononuclear cells (PBMC) of Borrelia positive patients. Data points obtained from the same donor with the iSpot Lyme assay and conventional ELISPOT assay are connected by a line. Each data point represents the mean spot forming unit (SFU) of triplicate antigen-stimulated wells minus the mean SFU of the corresponding medium control wells. A non-parametric Mann-Whitney $U$ test was used to compare the matched results with a $p$-value of $<0.05$ considered statistically significant. (B) Representative well images for test results obtained from one healthy control run in triplicate and $(\mathbf{C})$ from a Borrelia positive patient run in triplicate. (D) Size distribution of IFN- $\gamma$ ELISPOTs obtained from the iSpot Lyme assay $v s$. the conventional ELISPOT assay, as specified by the closed and open circles, respectively.

A

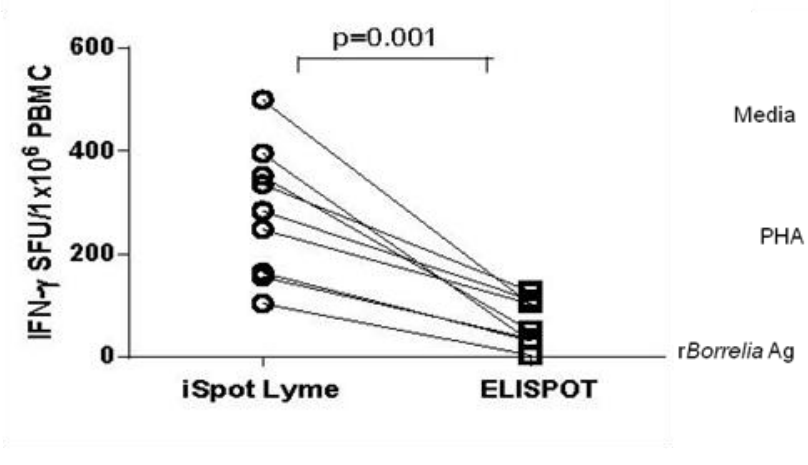

C

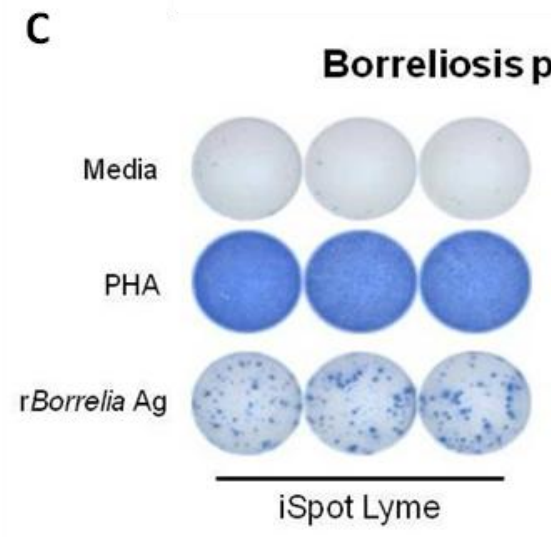

B Borreliosis negative donor
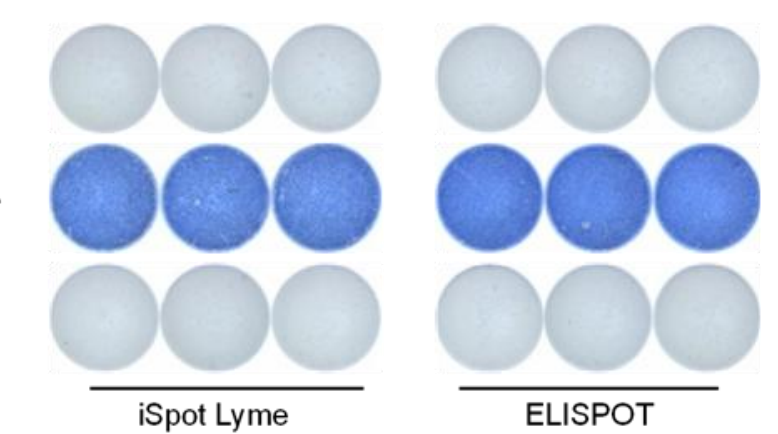

D

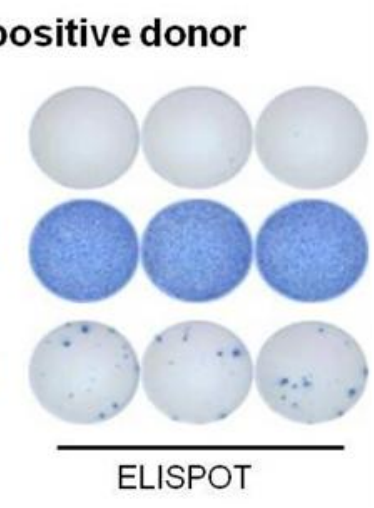

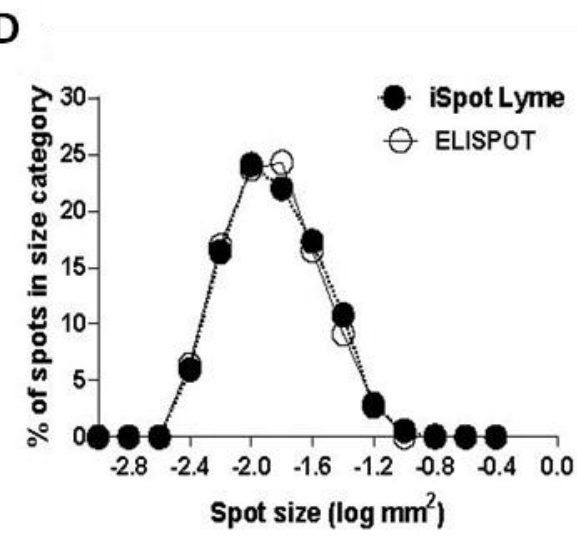


Figure 2. Evaluation of the iSpot Lyme assay as a diagnostic test. (A) The results of iSpot Lyme assays performed on 80 healthy controls (HC), 25 clinically diagnosed Lyme disease patients (LD) and 23 non-Lyme patients (NLP) are shown. Each symbol represents the mean SFU obtained in triplicate rBorrelia-stimulated wells of a test subject after subtraction of the mean SFU in triplicate medium control wells. Non-parametric Mann-Whitney U test was used to compare the results from LD vs. HC and LD vs. NLP. A p-value $<0.05$ was considered statistically significant. The dotted line represents the cutoff value for positivity at 25 SFU. (B) Receiver Operating Characteristics analysis was used to determine the sensitivity, specificity, positive predictive value (PPV), negative predictive value (NPV), area under the curve value (AUC) and cutoff value for the iSpot Lyme assay. (C) Receiver Operating Characteristics analysis was used to determine the sensitivity, specificity, PPV, NPV, AUC and cutoff value for the conventional ELISPOT assay.

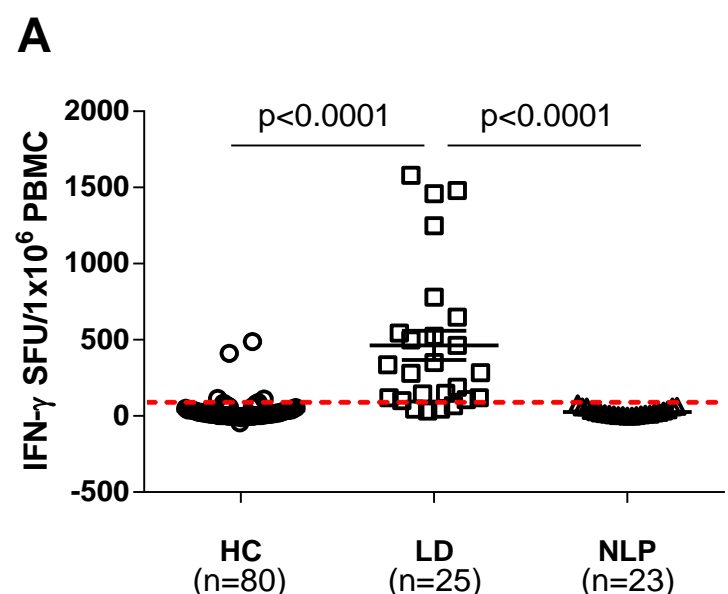

B

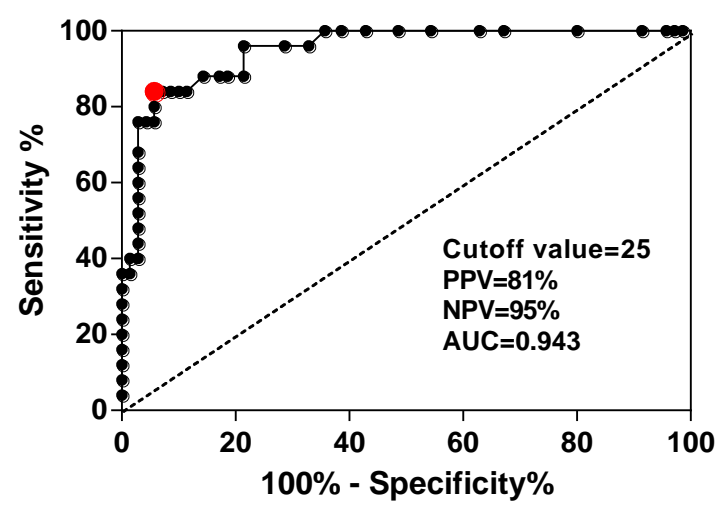

C

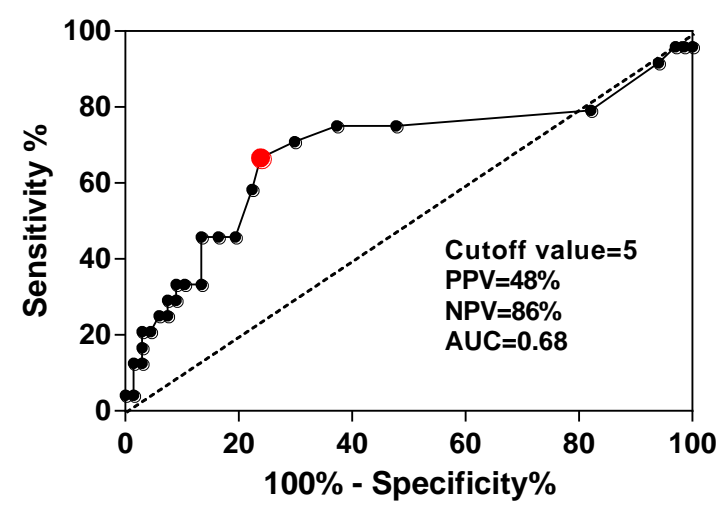




\subsection{Optimization and Validation of the iSpot Lyme Assay}

To determine the reliability of the iSpot Lyme assay as a routine laboratory test, we performed experiments to study its intra- and inter-assay precision. For the intra-assay precision studies, PBMC from five diagnosed Lyme patients were selected who displayed high, medium and low rBorrelia-triggered SFU values. Each of the PBMC samples were run in triplicate. As shown in Figure $3 \mathrm{~A}$, the coefficient of variation $(\mathrm{CV})$ among the triplicates ranged from $4.6 \%$ to $18.1 \%$ with a trend showing that an increase in $\mathrm{CV}$ is inversely proportional to SFU values. Inter-assay precision measurements were performed on 3 diagnosed Lyme patient PBMC samples on 5 consecutive days. To make sure that identical cell material was tested, that is, to avoid a biological variation of the sample itself due to blood collections at different times, we used cryopreserved PBMC samples from one blood draw and thawed one aliquot each day for this assay. The CV was 5.4\%, 5.9\% and 13.1\%, respectively (Figure 3B). These data suggested that the iSpot Lyme assay is a reliable test in terms of intra-assay and inter-assay precision.

Figure 3. Optimization and validation of the iSpot Lyme assay. (A) Intra-assay precision. Five PBMC samples with different rBorrelia-triggered SFU response levels were tested in triplicate wells each. Bars with the specified shades show the reactivity for the three individual wells, and the mean of the three. The coefficient of variation for the replicate wells was calculated. (B) Inter-assay precision. Cryopreserved PBMC aliquots of the specified three Lyme patients were tested for rBorrelia reactivity on five consecutive days. The coefficient of variation for inter assay variation was calculated. (C) Relationship between PBMC numbers plated in each well and the IFN- $\gamma$ SFU in a Borrelia positive subject, and (D), in a healthy control. Open symbols represent the mean of triplicate antigen-stimulated (treated) wells, the closed symbols represent the mean of the corresponding medium (control) wells. The Standard Deviation (SD) for the triplicate is smaller than the symbol when not visible. (E) Dose response curve for rBorrelia antigen-stimulated PBMC. (F) Correlation of the frequency of IFN- $\gamma$ secreting Borrelia-specific T cells assessed by the iSpot Lyme assay and the concentrations of IFN- $\gamma$ in the culture supernatant as measured by Bio-Plex suspension array. The nonparametric Spearman's test was used to determine the correlation. The results showed a $p$-value $<0.0001$.

A

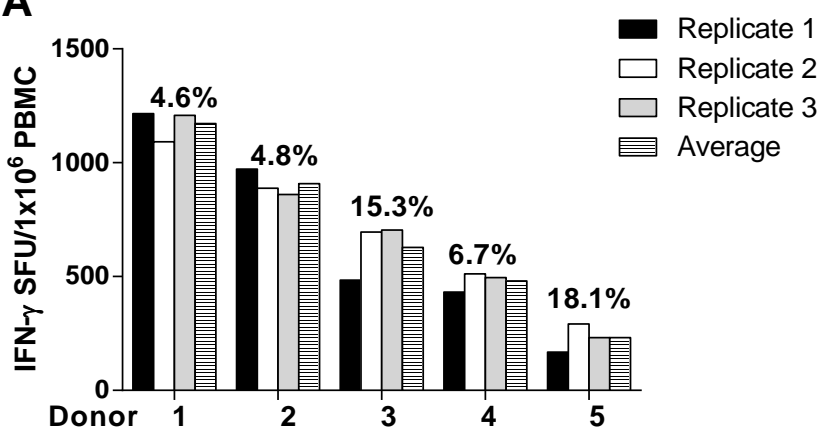

B

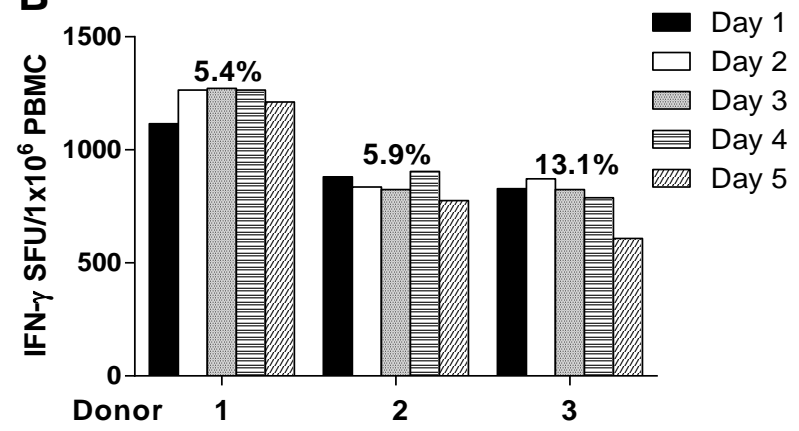


Figure 3. Cont.
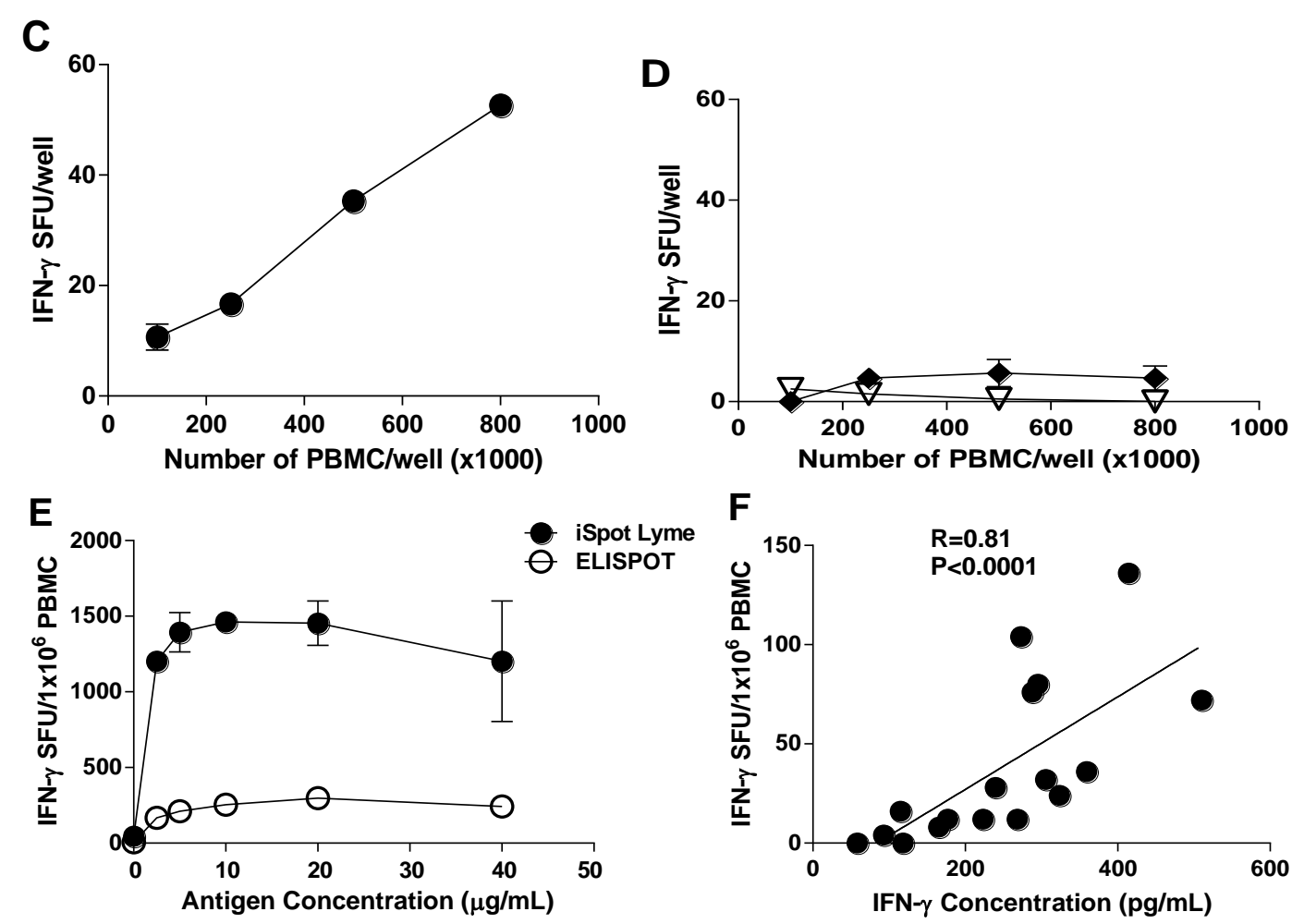

The results of an ELISPOT assay can be influenced by the PBMC numbers plated. We therefore tested the relationship between PBMC numbers plated per well, and the rBorrelia-induced IFN- $\gamma$ SFU per well. A linear relationship was seen (Figure 3C), similar to observations made in other antigen-specific ELISPOT systems [39]. The data show that variability in the rBorrelia-induced SFU count will depend on the accuracy of cell counting when adjusting the PBMC concentration, and the precision of pipetting. With 250,000 PBMC plated per well, the variability will be directly proportional to the magnitude of such imprecisions. When PBMC of healthy controls were plated in increasing numbers, the IFN- $\gamma$ spot numbers did not increase with or without $\mathrm{rBorrelia}$ antigen included in the test system (Figure 3D), suggesting that the IFN- $\gamma$ spots are produced specifically by Borrelia-reactive $\mathrm{T}$ cells in response to ex vivo restimulation by $\mathrm{r}$ Borrelia antigen.

The antigen concentration affects activation of the specific $\mathrm{T}$ cells [40]. We therefore tested rBorrelia antigen in serial dilution in Borrelia positive donors. As shown in Figure 3E, when the antigen concentration was increased, the numbers of SFU also increased initially rapidly but reached a plateau value starting at $10 \mu \mathrm{g} / \mathrm{mL}$. The $\mathrm{rBorrelia}$ dose-response curve was very similar for the iSpot Lyme assay and the conventional ELISPOT assay; however, the SFU values were significantly higher in the iSpot Lyme assay. The rBorrelia concentration of $10 \mu \mathrm{g} / \mathrm{mL}$ in the iSpot Lyme Test kit, therefore, is safely in the plateau of the dose response curve and inaccuracies in pipetting the antigen are a low risk factor for the test. These results also confirm that the iSpot Lyme assay is superior to the conventional ELISPOT approach for the detection of low frequencies of Borrelia-specific effector/memory T cells.

Assay validation includes the determination of accuracy as established by using an independent readout system for verifying assay results [41]. Therefore, we studied the correlation between IFN- $\gamma$ SFU numbers as established by iSpot Lyme assay, and the concentration of soluble IFN- $\gamma$ in the 
culture supernatants as measured by Bio-Plex method. As shown in Figure 3F, the results of the iSpot Lyme assay were closely correlated to the IFN- $\gamma$ concentrations as measured by Bio-Plex method $(\mathrm{R}=$ 0.81 and $p<0.0001)$. Overall, the validation results showed that the iSpot Lyme assay is a reliable and sensitive test for detecting Borrelia-specific T cells with the potential application in clinical laboratory diagnosis for Borrelia infection and Lyme disease.

\subsection{Comparisons between the iSpot Lyme Assay and Lyme Western Blot Assay}

The Western Blot assay has been used conventionally in aiding the clinical diagnosis of Lyme disease [13]. Lyme patients can be classified into two groups according to their serum antibody reactivity to Borrelia antigens [24]. Patients whose test positive in Western Blot are seropositive Lyme patients. Accordingly, Lyme patients who do not have detectable antibody levels are defined as seronegative patients. To compare the sensitivity of the iSpot Lyme assay with a Western Blot assay, we performed a study with 23 clinically diagnosed Lyme patients. The Western Blot assay showed $30 \%$ positivity in this group of patients whereas the iSpot Lyme assay and conventional ELISPOT showed $84 \%$ and 50\% positivity, respectively (Figure 4A). In this study, all five patients with positive Western Blot results were also positive for the iSpot Lyme assay. However, in another study we performed, we found that some patients who were positive on their Western Blot assay, had negative results with the iSpot Lyme assay (Data not shown). This discrepancy between the iSpot Lyme assay and the Western Blot assay could be attributed to several factors. One possibility is that there is a dissociation between humoral and cellular immunity to Borrelia infection [24]. The other possibility is that the positive results of Western Blot could be false positive as reported by a recent study in which $27.5 \%$ of patients who were tested based on suspicion of Lyme disease were found to have a false positive IgM Western Blot result [14]. In addition, we cannot exclude the possibility that $\mathrm{T}$ cell responses in some patients may be compromised due to use of immunosuppressive agents or other clinical conditions.

Figure 4. Comparison of sensitivity and specificity for detecting Borrelia infection via measuring $\mathrm{T}$ cell immunity by ELISPOT vs. serum antibodies by Western Blot. (A) Lyme ELISPOT assays and Western Blot assay were performed on PBMC and serum of 22 clinically diagnosed Borrelia patients. The percentage of individuals who scored positive for each assay is shown. (B) Cross-reactivity was assessed in 23 subjects with other clinical conditions, as defined in Materials and Methods, using the iSpot Lyme, conventional ELISPOT and Western Blot assay.
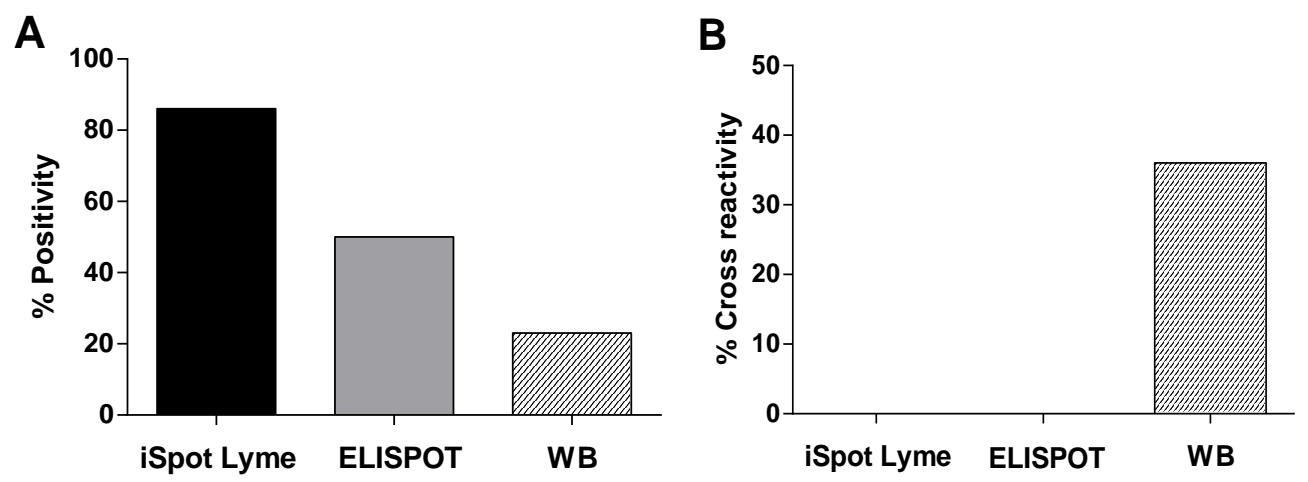
In summary, these results demonstrate firstly, that the Lyme ELISPOT assay is superior to the Western Blot assay in terms of sensitivity for detecting the underlying Borrelia infection. Secondly, the data suggests that there is a dissociation between the magnitude of the humoral and the T cell-mediated cellular immune response in Borrelia infection. Thirdly, the data implies that the iSpot Lyme assay may help identify Borrelia infected individuals when the serology-based diagnostic fails to do so.

In addition to the low sensitivity, the Western Blot assay also has relatively low specificity providing frequently false positive results including cross-reactivity with other clinical conditions such as other infectious diseases and autoimmune diseases [14]. To test the cross-reactivity for both the Western Blot assay and the Lyme ELISPOT assay (iSpot Lyme and conventional), 23 non-Lyme patients from low risk areas of Borrelia infection were studied. As shown in Figure 4B, the Western Blot assay gave $36 \%$ false positive results whereas both the conventional ELISPOT and iSpot Lyme assay did not have any cross-reactivity in the group of subjects studied. Therefore, Lyme ELISPOT assays, in particular the iSpot Lyme assay, are not only more sensitive but also more specific than the standard Western Blot serodiagnostic test for identifying Lyme disease and Borrelia infection. Due to the apparent prevalence of either humoral or cellular immunity in infected individuals, it is conceivable that the combination of the iSpot Lyme assay with Western Blot assay would further increase the sensitivity of Lyme disease diagnosis. Studies are ongoing in our laboratory to explore if the iSpot Lyme approach could be used to monitor disease progression and treatment of Lyme disease.

\section{Conclusions}

An enhanced T cell-based immunospot assay for Lyme disease was developed and validated. This iSpot Lyme assay can be used to study the T cell response elicited by Borrelia infection, which bridges the gap between the ability to detect humoral immunity and cellular immunity to Borrelia infection in Lyme disease. It may be a helpful laboratory diagnostic test for Lyme disease, especially for seronegative Lyme patients. Since serodiagnostic methods of Borrelia infection frequently provide false positive results, this $\mathrm{T}$ cell-based diagnostic test may help in confirming a Lyme diagnosis. A comprehensive evaluation of both antibody response and $\mathrm{T}$ cell response to Borrelia infection will provide new insights into the pathogenesis, diagnosis, treatment and monitoring the progress of Lyme disease.

\section{Acknowledgments}

We would like to acknowledge Andi M. Delong and Barbara M. Tigges for their technical assistance, Jodi M. Branstad for coordination and donor recruitment, and Delila J. Peri for editing the manuscript.

\section{Conflicts of Interest}

C.J., D.R.R., and G.H.K are employed by Pharmasan Laboratory that develops and offers diagnostic tests. P.V.L is employed by CTL which specializes on ELISPOT-based immune diagnostic assays.

\section{References}

1. Burgdorfer, W.; Barbour, A.G.; Hayes, S.F.; Benach, J.L.; Grunwaldt, E.; Davis, J.P. Lyme disease-a tick-borne spirochetosis? Science 1982, 216, 1317-1319. 
2. Steere, A.C. Lyme disease. N. Engl. J. Med. 2001, 345, 115-125.

3. Steere, A.C.; Coburn, J.; Glickstein, L. The emergence of Lyme disease. J. Clin. Invest. 2004, 113, 1093-1101.

4. Centers for Disease Control and Prevention. Reported Cases of Lyme Disease by Year, United States, 2002-2011. Available online: http://www.cdc.gov/lyme/stats/chartstables/casesbyyear.html (accessed on 1 July 2013).

5. Young, J.D. Underreporting of Lyme disease. N. Engl. J. Med. 1998, 338, 1629.

6. Gross, D.M.; Huber, B.T. Cellular and molecular aspects of Lyme arthritis. Cell. Mol. Life Sci. 2000, 57, 1562-1569.

7. Lim, L.C.; England, D.M.; DuChateau, B.K.; Glowacki, N.J.; Schell, R.F. Borrelia burgdorferi-specific T lymphocytes induce severe destructive Lyme arthritis. Infect. Immun. 1995, 63, 1400-1408.

8. Chandra, A.; Wormser, G.P.; Klempner, M.S.; Trevino, R.P.; Crow, M.K.; Latov, N.; Alaedini, A. Anti-neural antibody reactivity in patients with a history of Lyme borreliosis and persistent symptoms. Brain Behav. Immun. 2010, 24, 1018-1024.

9. Ramesh, G.; Benge, S.; Pahar, B.; Philipp, M.T. A possible role for inflammation in mediating apoptosis of oligodendrocytes as induced by the Lyme disease spirochete Borrelia burgdorferi. J. Neuroinflammation 2012, 9, 72 .

10. Aucott, J.; Morrison, C.; Munoz, B.; Rowe, P.C.; Schwarzwalder, A.; West, S.K. Diagnostic challenges of early Lyme disease: lessons from a community case series. BMC. Infect. Dis. 2009, $9,79$.

11. Dattwyler, R.J.; Volkman, D.J.; Halperin, J.J.; Luft, B.J.; Thomas, J.; Golightly, M.G. Specific immune responses in Lyme borreliosis. Characterization of $\mathrm{T}$ cell and B cell responses to Borrelia burgdorferi. Ann. N. Y. Acad. Sci. 1988, 539, 93-102.

12. Krause, A.; Burmester, G.R.; Rensing, A.; Schoerner, C.; Schaible, U.E.; Simon, M.M.; Herzer, P.; Kramer, M.D.; Wallich, R. Cellular immune reactivity to recombinant OspA and flagellin from Borrelia burgdorferi in patients with Lyme borreliosis. Complexity of humoral and cellular immune responses. J. Clin. Invest. 1992, 90, 1077-1084.

13. Aguero-Rosenfeld, M.E.; Wang, G.; Schwartz, I.; Wormser, G.P. Diagnosis of lyme borreliosis. Clin. Microbiol. Rev. 2005, 18, 484-509.

14. Seriburi, V.; Ndukwe, N.; Chang, Z.; Cox, M.E.; Wormser, G.P. High frequency of false positive IgM immunoblots for Borrelia burgdorferi in clinical practice. Clin. Microbiol. Infect. 2012, 18, 1236-1240.

15. Brown, S.L.; Hansen, S.L.; Langone, J.J. Role of serology in the diagnosis of Lyme disease. JAMA 1999, 282, 62-66.

16. Brunner, M. New method for detection of Borrelia burgdorferi antigen complexed to antibody in seronegative Lyme disease. J. Immunol. Methods. 2001, 249, 185-190.

17. Tylewska-Wierzbanowska, S.; Chmielewski, T. Limitation of serological testing for Lyme borreliosis: evaluation of ELISA and western blot in comparison with PCR and culture methods. Wien. Klin. Wochenschr. 2002, 114, 601-605. 
18. Forsberg, P.; Ernerudh, J.; Ekerfelt, C.; Roberg, M.; Vrethem, M.; Bergstrom, S. The outer surface proteins of Lyme disease borrelia spirochetes stimulate $\mathrm{T}$ cells to secrete interferon-gamma (IFN-gamma): Diagnostic and pathogenic implications. Clin. Exp. Immunol. 1995, 101, 453-460.

19. Horowitz, H.W.; Pavia, C.S.; Bittker, S.; Forseter, G.; Cooper, D.; Nadelman, R.B.; Byrne, D.; Johnson, R.C.; Wormser, G.P. Sustained cellular immune responses to Borrelia burgdorferi: Lack of correlation with clinical presentation and serology. Clin. Diagn. Lab. Immunol. 1994, 1, 373-378.

20. Nordberg, M.; Forsberg, P.; Nyman, D.; Skogman, B.H.; Nyberg, C.; Ernerudh, J.; Eliasson, I.; Ekerfelt, C. Can ELISPOT be applied to a clinical setting as a diagnostic utility for Neuroborreliosis? Cells 2012, 1, 153-167.

21. Aguero-Rosenfeld, M.E.; Nowakowski, J.; Bittker, S.; Cooper, D.; Nadelman, R.B.; Wormser, G.P. Evolution of the serologic response to Borrelia burgdorferi in treated patients with culture-confirmed erythema migrans. J. Clin. Microbiol. 1996, 34, 1-9.

22. Dressler, F.; Yoshinari, N.H.; Steere, A.C. The T-cell proliferative assay in the diagnosis of Lyme disease. Ann. Intern. Med. 1991, 115, 533-539.

23. Lastavica, C.C.; Snydman, D.R.; Schenkein, D.P.; Berardi, V.P.; Pariser, K.M. Demonstration of Borrelia burgdorferi in a patient with chronic Lyme arthritis. Zentralbl. Bakteriol. Mikrobiol. Hyg. A 1986, 263, 288.

24. Dattwyler, R.J.; Volkman, D.J.; Luft, B.J.; Halperin, J.J.; Thomas, J.; Golightly, M.G. Seronegative Lyme disease. Dissociation of specific T- and B-lymphocyte responses to Borrelia burgdorferi. N. Engl. J. Med. 1988, 319, 1441-1446.

25. Hilton, E.; Tramontano, A.; DeVoti, J.; Sood, S.K. Temporal study of immunoglobin M seroreactivity to Borrelia burgdorferi in patients treated for Lyme borreliosis. J. Clin. Microbiol. 1997, 35, 774-776.

26. Kalish, R.A.; McHugh, G.; Granquist, J.; Shea, B.; Ruthazer, R.; Steere, A.C. Persistence of immunoglobulin $\mathrm{M}$ or immunoglobulin $\mathrm{G}$ antibody responses to Borrelia burgdorferi 10-20 years after active Lyme disease. Clin. Infect. Dis. 2001, 33, 780-785.

27. Hagen, J.; Houchins, J.P.; Kalyuzhny, A.E. Combining ELISPOT and ELISA to measure amounts of cytokines secreted by a single cell. In Handbook of ELISPOT: Methods and Protocols, Methods in Molecular Biology, 2nd ed.; Kalyuzhny, A., Ed.; Springer Science+Business Media, LLC: New York, NY, USA, 2012; Volume 792, pp.115-122.

28. Lehmann, P.V.; Zhang, W. Unique Strengths of ELISPOT for T Cell Diagnostics. In Handbook of ELISPOT: Methods and Protocols, Methods in Molecular Biology, 2nd ed.; Kalyuzhny, A., Ed.; Springer Science+Business Media, LLC: New York, NY, USA, 2012; Volume 792, pp. 3-23.

29. Tary-Lehmann, M.; Hamm, C.D.; Lehmann, P.V. Validating Reference Samples for Comparison in a Regulated ELISPOT Assay. In Validation of Cell-Based Assays in the GLP Setting: A Practical Guide, 1st ed.; Prabhakar, U., Kelley, M., Eds.; John Wiley \& Sons, Ltd.: West Sussex, UK, 2008; pp. 127-146.

30. Lalvani, A.; Pathan, A.A.; Durkan, H.; Wilkinson, K.A.; Whelan, A.; Deeks, J.J.; Reece, W.H.; Latif, M.; Pasvol, G.; Hill, A.V. Enhanced contact tracing and spatial tracking of Mycobacterium tuberculosis infection by enumeration of antigen-specific T cells. Lancet 2001, 357, 2017-2021. 
31. Lalvani, A. Counting antigen-specific $\mathrm{T}$ cells: A new approach for monitoring response to tuberculosis treatment? Clin. Infect. Dis. 2004, 38, 757-759.

32. Centers for Disease Control and Prevention. Signs and Symptoms of Lyme Disease, United States. 2013. Available online: http://www.cdc.gov/lyme/signs_symptoms/index.html (accessed on 1 July 2013).

33. Wormser, G.P.; Dattwyler, R.J.; Shapiro, E.D.; Halperin, J.J.; Steere, A.C.; Klempner, M.S.; Krause, P.J.; Bakken, J.S.; Strle, F.; Stanek, G.; et al. The clinical assessment, treatment, and prevention of lyme disease, human granulocytic anaplasmosis, and babesiosis: Clinical practice guidelines by the Infectious Diseases Society of America. Clin. Infect. Dis. 2006, 43, 1089-1134.

34. Gross, D.M.; Forsthuber, T.; Tary-Lehmann, M.; Etling, C.; Ito, K.; Nagy, Z.A.; Field, J.A.; Steere, A.C.; Huber, B.T. Identification of LFA-1 as a candidate autoantigen in treatment-resistant Lyme arthritis. Science 1998, 281, 703-706.

35. Widhe, M.; Jarefors, S.; Ekerfelt, C.; Vrethem, M.; Bergstrom, S.; Forsberg, P.; Ernerudh, J. Borrelia-Specific interferon-gamma and interleukin-4 secretion in cerebrospinal fluid and blood during Lyme borreliosis in humans: association with clinical outcome. J. Infect. Dis. 2004, 189, 1881-1891.

36. Widhe, M.; Skogman, B.H.; Jarefors, S.; Eknefelt, M.; Enestrom, G.; Nordwall, M.; Ekerfelt, C.; Croner, S.; Bergstrom, S.; Forsberg, P.; et al. Up-regulation of Borrelia-specific IL-4- and IFN-gamma-secreting cells in cerebrospinal fluid from children with Lyme neuroborreliosis. Int. Immunol. 2005, 17, 1283-1291.

37. Zhang, W.; Tary-Lehmann, M. Objective, User-Independent ELISPOT Data Analysis Based on Scientifically Validated Principles. In Validation of Cell-Based Assays in the GLP Setting: A Practical Guide, 1st ed.; Uma Prabhakar, Marian Kelley, Eds.; John Wiley \& Sons, Ltd.: West Sussex, UK, 2008; pp. 126-147.

38. Ekerfelt, C.; Forsberg, P.; Svenvik, M.; Roberg, M.; Bergstrom, S.; Ernerudh, J. Asymptomatic Borrelia-seropositive individuals display the same incidence of Borrelia-specific interferon-gamma (IFN-gamma)-secreting cells in blood as patients with clinical Borrelia infection. Clin. Exp. Immunol. 1999, 115, 498-502.

39. Zhang, W.; Caspell, R.; Karulin, A.Y.; Ahmad, M.; Haicheur, N.; Abdelsalam, A.; Johannesen, K.; Vignard, V.; Dudzik, P.; Georgakopoulou, K.; et al. ELISPOT assays provide reproducible results among different laboratories for T-cell immune monitoring--even in hands of ELISPOT-inexperienced investigators. J. Immunotoxicol. 2009, 6, 227-234.

40. Hesse, M.D.; Karulin, A.Y.; Boehm, B.O.; Lehmann, P.V.; Tary-Lehmann, M. A T cell clone's avidity is a function of its activation state. J. Immunol. 2001, 167, 1353-1361.

41. Fardy, J. Evaluation of Diagnositc Tests. In Methods of Molecular Biology, Clinical Epidemiology; Parfrey, P., Barrett, B., Eds.; Humana Press: Totowa, NJ, USA, 2009; Volume 473, pp. 127-136.

(C) 2013 by the authors; licensee MDPI, Basel, Switzerland. This article is an open access article distributed under the terms and conditions of the Creative Commons Attribution license (http://creativecommons.org/licenses/by/3.0/). 